\title{
Quality and Capacity Analysis of Molecular Communications in Bacterial Synthetic Logic Circuits
}

\author{
Daniel P. Martins, Student Member, IEEE, Michael Taynnan Barros, Member, IEEE, \\ and Sasitharan Balasubramaniam, Senior Member, IEEE
}

\begin{abstract}
Synthetic logic circuits have been proposed as potential solutions for theranostics of biotechnological problems. One proposed model is the engineering of bacteria cells to create logic gates, and the communication between the bacteria populations will enable the circuit operation. In this paper, we analyse the quality of bacteria-based synthetic logic circuit through molecular communications that represent communication along a bus between three gates. In the bacteria-based synthetic logic circuit, the system receives environmental signals as molecular inputs and will process this information through a cascade of synthetic logic gates and free diffusion channels. We analyse the performance of this circuit by evaluating its quality and its relationship to the channel capacity of the molecular communications links that interconnect the bacteria populations. Our results show the effect of the molecular environmental delay and molecular amplitude differences over both the channel capacity and circuit quality. Furthermore, based on these metrics we also obtain an optimum region for the circuit operation resulting in an accuracy of $80 \%$ for specific conditions. These results show that the performance of synthetic biology circuits can be evaluated through molecular communications, and lays the groundwork for combined systems that can contribute to future biomedical and biotechnology applications.
\end{abstract}

Index Terms-Synthetic logic circuits, Molecular communications, Engineered bacteria.

\section{INTRODUCTION}

$\mathbf{R}$ ECENT progress in synthetic biology has seen a number of new technologies developed for engineering biological cells utilizing concepts from electrical engineering and computer science [1]-[5]. Unprecedented applications have emerged from synthetic engineering of cells, leading to novel approaches in the fields of biotechnology, medicine, as well as pharmaceutical science [4], [6]-[8]. Example applications have enabled researchers to engineer eukaryotic as well as prokaryotic cells (e.g., animal cells and bacteria, respectively) that can sense enzymes secreted from cancerous cells or treatment of systemic diseases such as Inflammatory Bowel Disease [7]. The multi-disciplinary approach combines principles from both electronics engineering and molecular biology to design the building blocks, where their combination can

Daniel P. Martins and Michael Taynnan Barros are with the Telecommunication Software \& Systems Group (TSSG), Waterford Institute of Technology (WIT), Ireland. E-mail: dpmartins@tssg.org.

Sasitharan Balasubramaniam is with the Telecommunication Software \& Systems Group (TSSG), Waterford Institute of Technology (WIT), Ireland and with the Faculty of Information Technology and Communication Sciences, Tampere University, Finland. Email: sasi.bala@tut.fi.

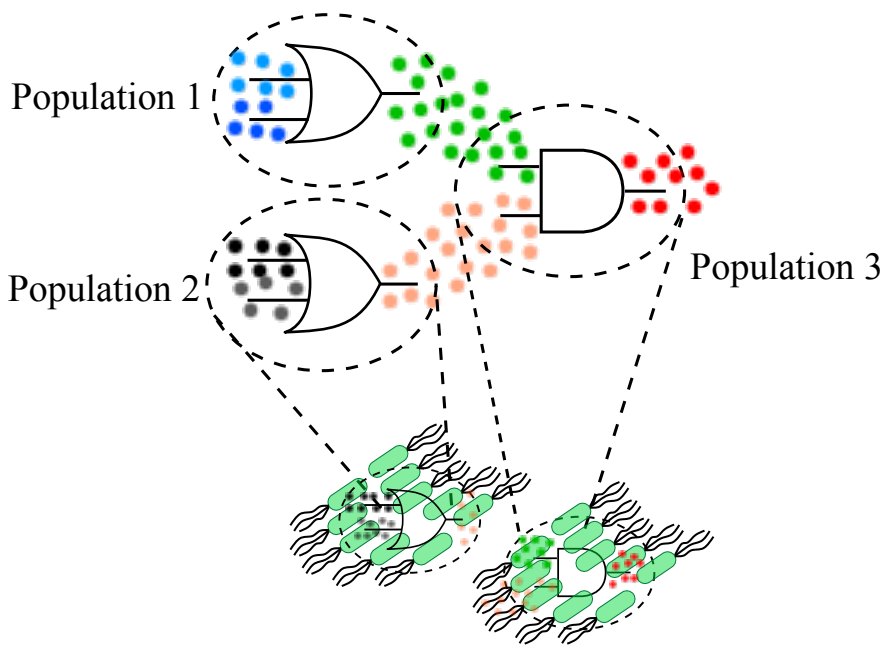

Fig. 1. Illustration of bacteria populations engineered to perform logical operations. For these systems, the input signals are molecules from the environment that are absorbed by the engineered bacteria.

lead to complex synthetic structures such as logic gates [9][13]. However, complex circuits are still an obstacle as the current logic structures are often designed using a single logic gate or simple combinations between them [3].

Bacterial cells have been widely used to develop synthetic circuits due to the extensive knowledge about the DNA plasmids, and in particular in genome editing to design synthetic circuits that can lead to specialised functions [6]. Simple logic gates, to toggle switches, as well as oscillators, have already been developed by engineering both single cell and bacterial population [10], [14]. An overall representation of synthetic logic circuits constructed from bacteria is presented in Figure 1. These circuits are usually associated with controlling the bacteria's communication behaviour. As an example, researchers were able to engineer Boolean logic gates by controlling the communications between four Escherichia coli populations [15]. With the recent advancements in animal microbiome research, engineered bacteria are being considered as potential biomedical agents that combine diagnostic and individualised treatment (i.e., theranostic) for systemic diseases inside the human microbiome [6], [7], [10]. Specifically, the communication molecules produced by engineered bacteria are the effectors for these applications.

Due to the importance of molecular signals that can be used for the development of biomedical theranostics, researchers 
are devoting their attention to understand also how they can be engineered. This new emerging subject is known as molecular communications [16]-[20]. This field concentrates on the development of artificial communications systems for biological devices at the nanoscale [16], [17], [21]-[25]. As it is inspired from natural systems, molecular communications systems require the engineering of molecular exchange that can be developed in a biological cell, and this could range from the engineering of cells for ion transfer to design networked communications among nanosystems [16], [17], [19], [20], [25]-[27]. As an example, bacteria-based molecular communications has been proposed to emit molecular signals in a networked model in order to produce jamming signals that interfere with another populations communications [28]-[30].

In this paper, we propose the use of bacteria-based molecular communication systems to evaluate the performance of synthetic logic circuits inside a multi-compartment capsule that contains three bacteria populations (each one is representing a different Boolean logic gate). The logic circuit is then built using the communications among the engineered bacteria confined in each compartment of the chamber. The design of this synthetic logic circuit prevents the engineered bacteria from spreading into the environment while allowing different logic operations to be performed depending on different molecular inputs. The confined chambers have two roles for this bacteria-based molecular communications system. First it limits the bacteria movement, where no organisms will be able to enter or leave the chamber. Second, the chamber prevents larger molecules from diffusing between the compartments. Therefore, these features limit the impact of unwanted effects in the system (e.g., exogenous bacteria hijacking the system or high communications noise due to the presence of unwanted molecules). Based on these assumptions, we focus our investigation on the impact of the noise generated by the engineered bacteria during their production of quorum sensing molecules.

The design principles considered in this paper follows similar approaches for other bacteria-based synthetic circuits. For example, toggle switches, logic gates, biosensors and programmable full-adder were designed using the bacteria signalling molecules as the activator and repressor of gene expression [11], [31]-[34]. Specifically, our bacteria-based synthetic logic circuit is inspired by a similar work in [15], where three Escherichia coli populations carried the same NOR gate and another carried the buffer. These bacterial populations were combined in different spatial arrangements to create 16 twoinput Boolean logic gates [15]. Their work served as a proofof-concept for using quorum sensing signalling as chemical 'wires' to interconnect the bacterial populations' logic gates. However, the work presented in this paper does not concentrate on the versatile combination of different synthetic logic gates, but instead on the analysis of molecular information between the gates to determine the quality of the circuit's performance.

Our molecular communications analysis is focused on showing the quality of the proposed bacteria-based synthetic logic circuit in conjunction with the evaluation of the capacity of the channels that interconnect the bacteria populations. Similar to digital circuits that can become faulty due to unreliable interconnection bus [35], [36], we investigate the effect of the free diffusion interconnections and the impact on the quality of the proposed circuit design. The proposed bacteria-based synthetic circuit is capable of sensing environmental variations and actuating accordingly in response to the measurements. This circuit composed of a two-layer synthetic logic circuit that will process the input molecular signals and output the required signal. For this paper, we do not intend to provide further specification on the sensing and actuating functionalities, but only on the communication performance. The main contributions of this paper are

- Development of a bacteria-based molecular communications end-to-end model that can be used to analyse the reliability performance of a synthetic logic circuit. We use three bacteria populations to create a synthetic logic circuit that will perform a cascade of logical operations at the molecular level.

- Computation of quality performance metrics for the synthetic logic circuit operation considering different scenarios. We analyse the unreliable nature of bacteriabased molecular communications systems that can disrupt the operation of the synthetic circuit, affecting its accuracy and precision.

- Analysing the channel capacity for the bacteria-based synthetic logic circuit. Using molecular communications theory, we show how different combination of parameters will affect the reliability of a circuit and how they are associated with the optimum channel capacity for the system.

The rest of the paper is organised as follows. In Section II, we describe the bacteria-based synthetic logic circuit, from both biological and communications engineering perspectives, and how they will be utilized to perform logic operations based on a set of molecular input signals. The communication model that represents the exchange of molecular information between the bacteria populations is presented in Section III. Section V presents the application of this model to determine the quality and the channel capacity for the proposed synthetic circuit. Lastly, in Section VI we present our conclusions.

\section{SySTEM DESIGN}

The proposed circuit is a combination of three synthetic logic gates (one OR and two AND gates) that use a set of input molecular signals (see Figure 2). Although this paper only concentrates on this specific circuit, further analysis can be established for other logic gate combinations and circuit size. Each gate, represented in Figure 2(a), is composed of a bacteria population and is situated in a compartment, and is interconnected by free diffusion molecular communications links. These bacteria populations are placed inside a compartmented capsule with isolated chambers. For example, compartment A (OR gate) and B (AND gate) only communicates with the third compartment C (AND gate), through a filter wall (see Figure 2(b)). This thin membrane can be built using an organic compound such as Polydimethylsiloxane (PDMS), to only allow the molecules but not the bacteria to flow from chambers A and B to C [37]. A general representation of this filtering process is depicted in Figure 3. 


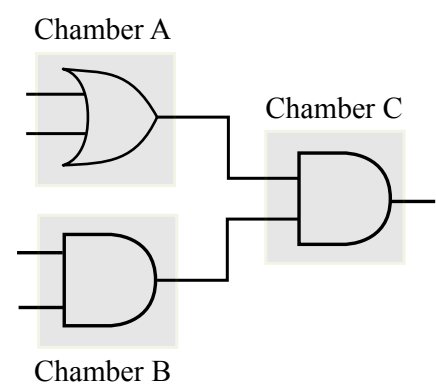

(a) Digital circuit representation of the system.

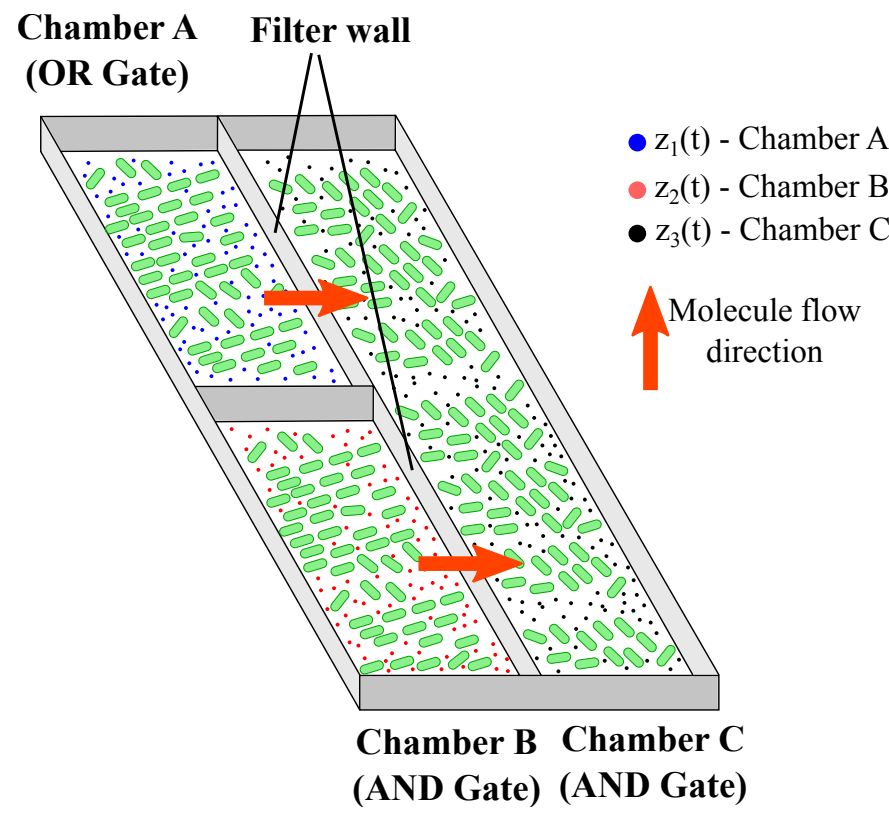

(b) Proposed system's physical design.

Fig. 2. Illustration of the proposed bacteria-based synthetic logic circuit. Each bacteria population is assigned to a compartment, becoming isolated from each other. The molecules produced at chambers A or B access chamber $\mathrm{C}$ by passing through a filter wall.

We limit the growth of the bacteria populations to fit the finite dimensions of each chamber. This population control can be achieved by applying antibiotics or probiotics, which will lower or increase their population density, respectively [38], [39]. Therefore, we consider that the bacteria population densities are constant. Based on this assumption, our analysis on the circuit's quality and the communications capacity is focused on the maximum bacteria population density.

The circuit operation can be described in two steps. First, each bacterium senses the molecular environmental signals $w_{i}\left(t-\tau_{i}\right)$ and starts the quorum sensing process to produce molecules that will toggle their natural switches (see [30] for further description of this process). The mathematical description of the molecular environmental signal $w_{i}\left(t-\tau_{i}\right)$ can be found in Section III-A. The molecular environmental delays $\tau_{1}, \tau_{2}, \tau_{3}$ and $\tau_{4}$ are produced by the different propagation times of each molecular input signal on the chamber's external medium. Second, all bacteria inside the chambers start to behave similarly to perform the desired logic operations. This process occurs for the first layer of the circuit. From

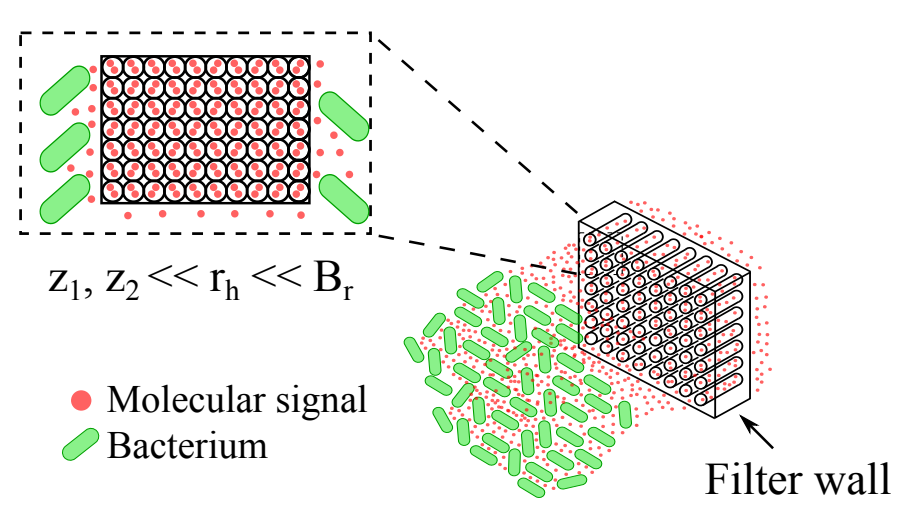

Fig. 3. Representation of the wall that separates the input gates from the output gate. This wall acts like a filter just allowing the molecular signal to pass through. For that purpose, the radius of the holes $r_{h}$, depicted in the expanded view of this Figure, must be greater than the molecule signal $\left(z_{1}(t)\right.$ and $\left.z_{2}(t)\right)$ sizes and lesser than the bacterium radius $B_{r}$.

an electronic circuit perspective, this synthetic circuit can be defined as an arrangement of logic gates and RC circuits [40]. This analogy allows us to investigate the quality of the system using the same metrics of a typical electronic circuit [41], [42].

\section{A. Quorum Sensing and Molecule Binding Process}

Bacteria can sense and respond to molecular signals that originate from other cells and environment, through a cellular communication process named quorum sensing [43]. This cell-to-cell communication process allows bacteria to display group behaviours through the controlled expression of genes, regulating many of their activities. Bacteria can survive harsh environments, produce virulent factors and form biofilms by using quorum sensing systems [43]. Each bacteria species uses specific molecules to perform this type of communication, and examples include autoinducers and N-Acyl homoserine lactone (AHL's). They also have specific transmembrane receptors to detect these signalling molecules, and examples include LuxN and CqsS [43]. These receptors bind quorum sensing molecules and trigger the expression of genes related to bacteria collective behaviours. Inside each bacterium cell, an initial cluster of genes (operon) will process the molecular signal originated from the transmembrane proteins and promote the second cluster of genes (effector) to transcribe messenger RNAs (mRNAs) [2], [44]. These molecules will be responsible for the translation of the genetic instruction into proteins that will trigger the group behaviours.

Since the first identification of this signalling mechanism, researchers have been proposing synthetic biology techniques that produce different responses for the sensed quorum sensing molecules [9]-[15]. They engineer new reception circuits in the bacteria, through genetic editing, in order to activate a response after sensing a certain concentration level that is tied to the number of individuals in a bacteria population [7]. The molecule binding process has also been applied for the design of molecular communications systems [45], [46]. In this paper, we focus on the use of engineered bacteria to perform logic operations through the use of quorum sensing molecules diffused in a closed space. 

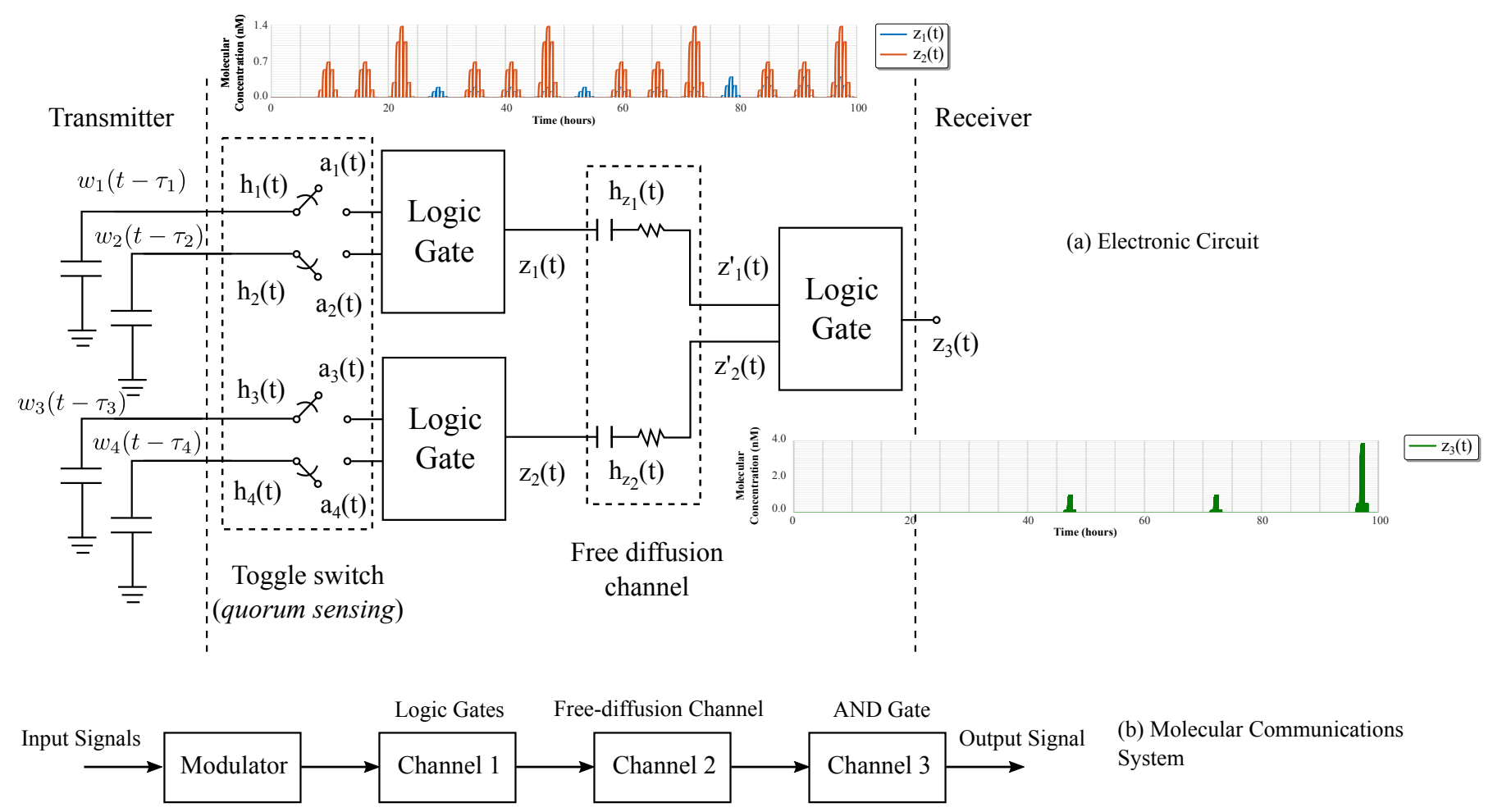

Fig. 4. Illustration of the proposed bacteria-based synthetic logic circuit. The four molecular input signals required to operate the system are processed by a cascade of synthetic logic gates and free diffusion channels. The natural toggle switches, based on the quorum sensing, are represented as On-Off systems and the free diffusion channels as an RC circuit. (a) Electronic circuit representation. (b) Molecular communications system representation.

TABLE I

TRUTH TABLE OF THE SYNTHETIC CIRCUIT CONSIDERED FOR THIS WORK. THE HIGH MOLECULAR SIGNAL LEVEL OUTPUT IS HIGHLIGHTED IN GRAY.

\begin{tabular}{ccccc}
\hline Input $_{1}$ & Input $_{\boldsymbol{2}}$ & Input $_{\boldsymbol{3}}$ & Input $_{\mathbf{4}}$ & Circuit Output \\
\hline 0 & 0 & 0 & 0 & 0 \\
0 & 0 & 0 & 1 & 0 \\
0 & 0 & 1 & 0 & 0 \\
0 & 0 & 1 & 1 & 0 \\
0 & 1 & 0 & 0 & 0 \\
0 & 1 & 0 & 1 & 0 \\
0 & 1 & 1 & 0 & 0 \\
0 & 1 & 1 & 1 & 1 \\
1 & 0 & 0 & 0 & 0 \\
1 & 0 & 0 & 1 & 0 \\
1 & 0 & 1 & 0 & 0 \\
1 & 0 & 1 & 1 & 1 \\
1 & 1 & 0 & 0 & 0 \\
1 & 1 & 0 & 1 & 0 \\
1 & 1 & 1 & 0 & 0 \\
1 & 1 & 1 & 1 & 1
\end{tabular}

\section{B. Synthetic Logic Gates}

A synthetic biology system is often described using rate reaction equations [45]. These are mathematical formulations that represent the change in the concentration of chemical substances during a given period. These equations are expressed in terms of the rate of production and consumption of those chemical substances and are described as follows

$$
\left[\mathrm{R}_{1}\right]+\left[\mathrm{R}_{2}\right] \underset{\mathrm{k}_{\mathrm{r}}}{\stackrel{\mathrm{k}_{\mathrm{f}}}{\rightleftharpoons}}[\mathrm{P}],
$$

where $\left[R_{1}\right]$ and $\left[R_{2}\right]$ are the two chemical substances that react to each other to produce the substance $[\mathrm{P}] ; k_{f}$ is the forward reaction rate, in other words, is the speed of $\left[R_{1}\right]$ and $\left[R_{2}\right]$ reaction to produce [P]; and $k_{r}$ is the reverse reaction rate, or the speed of $[P]$ degradation to form $\left[R_{1}\right]$ and $\left[R_{2}\right]$.

In this paper, we use rate reaction equations to model the activation of the first layer of the synthetic logic circuit and the production of the molecular output signals from the three synthetic logic gates. Therefore, we describe the natural switch activation (see Figure 4) using the following equations [11]

$$
\frac{d[A]}{d t}=\frac{\beta}{1+K_{B}^{\alpha}}-\gamma_{t}[A]
$$

and

$$
\frac{d[B]}{d t}=\frac{\beta}{1+K_{A}^{\alpha}}-\gamma_{t}[B]
$$

where $[A]$ and $[B]$ represents the molecular signal concentrations that trigger and suppresses the operation of the synthetic logic gate, respectively; $\alpha$ and $\beta$ are the repression constants, and the maximum production rates for both molecular signals $[A]$ and $[B] ; s_{A}$ and $s_{B}$ are the toggle switches' induction signals, $K_{A}=[A] /\left(1+\left(s_{A} / K\right)^{n_{t}}\right)$ and $K_{B}=[B] /(1+$ $\left.\left(s_{B} / K\right)^{n_{t}}\right)$ define the rates by which the molecular signals bind to the synthetic gate receptor, $K$ is a constant that defines the equilibrium of the chemical reactions involved in the production of a molecular signal, $\gamma_{t}$ is the decay constant for both molecular signals, and $n_{t}$ is the cooperativeness degree of the molecular signal with the synthetic gate receptor (this is also known as the Hill coefficient). 
After reaching the threshold required to toggle the bacterium's natural switches, the circuit will start to operate. The first two gates shown in Figure 4 are activated by molecular input signals $w_{i}(t)$, where $i=\{1,2,3,4\}$ are the molecular input's index, diffused from the environment into the synthetic logic circuit. The last logic gate is activated by the outputs $\left(z_{1}(t)\right.$ and $\left.z_{2}(t)\right)$ from the gates in the first layer, producing the molecular signal $z_{3}(t)$. Considering the specific circuit design (see Figure 4), this last output molecular signal will only have a high concentration signal for the cases highlighted in Table I. Similar to (2) and (3), we use rate reaction equations to describe the production of the molecular signals $z_{1}(t), z_{2}(t)$ and $z_{3}(t)$ by the synthetic logic gates [47]. Therefore, the OR gate is evaluated as

$$
\begin{aligned}
\frac{d[O R]}{d t}= & \frac{[C]^{n_{C}}}{K_{C}^{n_{C}}+[C]^{n_{C}}}+\frac{[D]^{n_{D}}}{K_{D}^{n_{D}}+[D]^{n_{D}}} \\
& -\gamma_{O R}[O R]+N_{O R}(t),
\end{aligned}
$$

where $K_{C}$ and $K_{D}$ define the rates (association constants) by which $[C]$ and $[D]$ bind to the OR gate receptors; $\gamma_{O R}$ is the decay constant for $[O R] ; n_{C}, n_{D}$ are the Hill coefficients; and $N_{O R}(t)$ is the noise resulting from the chemical reaction for this synthetic logic gate. This noise term is modelled as an Additive White Gaussian Noise - AWGN. The "whitening effect" of the molecular noise in quorum sensing systems was investigated by [48]. Quorum sensing production noise can be modelled as a short noise with a wideband spectrum [49]. Shot noise is often modelled as a Poisson process, but if its mean value is large enough (following the central limit theorem), this noise can be modelled as a Gaussian noise [50]. In this case, if there is a large forward reaction rate compared to the reverse reaction rate, see (1), the noise will spread their frequency spectra and approximate it to an Additive White Gaussian Noise [48]. Following this assumption, our circuit will produce the output molecular signals in higher quantity, which will also prevent reverse reaction in (1) that will lead to the original input molecules. The reaction in (1) for both forward and reverse process generates the noise that is represented as an AWGN noise. Similarly, the AND gate is evaluated as

$$
\begin{aligned}
\frac{d[A N D]}{d t}= & \frac{[E]^{n_{E}}}{K_{E}^{n_{E}}+[E]^{n_{E}}} \cdot \frac{[F]^{n_{F}}}{K_{F}^{n_{F}}+[F]^{n_{F}}} \\
& -\gamma_{A N D}[A N D]+N_{A N D}(t),
\end{aligned}
$$

where $K_{E}, K_{F}$ are the association constants for the signals $[E]$ and $[F] ; \gamma_{A N D}$ is the decay constants for $[A N D] ; n_{E}$ and $n_{F}$ are the Hill coefficients for these molecular signals; and $N_{A N D}(t)$ is the AWGN noise for these molecular output signals.

\section{Communications Model}

An overview representation of the proposed bacteria-based molecular communications model is depicted in Figure 4. This synthetic logic circuit can be viewed, from a communications perspective, as a combination of three channels (see Figure $4 \mathrm{~b}$ ), which processes the molecular signals $a_{i}\left(t-\tau_{i}\right), z_{1}(t)$, $z_{2}(t), z_{1}^{\prime}(t)$ and $z_{2}^{\prime}(t)$. In this paper, we do not investigate the effects of the aqueous medium over the molecular input signals as they will be generated by sources much larger than the bacteria-based synthetic logic circuit, allowing them to retain their characteristics. In the following, we provide a detailed description of the proposed model for the synthetic logic circuit.

\section{A. End-to-End Model}

We model the bacteria-based synthetic logic circuit as a finite 2D aqueous environment where the molecular signals produced in the chambers A and B will freely diffuse towards the chamber $\mathrm{C}$. The first layer of the synthetic circuit is activated by four molecular environmental signals, two different signals for each bacterial population, and is represented as

$$
w_{i}\left(t-\tau_{i}\right)=W_{i} \frac{\exp \left(\frac{-\left(t-\tau_{i}\right)^{2}}{2 \eta^{2}}\right)}{\sqrt{2 \pi \eta}},
$$

where $W_{i}$ is the pulse amplitude and $\eta$ is the spread of the pulse. These four signals will be propagated from the external environment to the synthetic logic circuit and will bind to the receptors inside each bacterium to activate the internal toggle switches (see Figure 4). This internal bacterium system triggering process will result in the On-Off-Keying (OOK) modulation of the molecular signals $w_{1}\left(t-\tau_{1}\right), w_{2}\left(t-\tau_{2}\right)$, $w_{3}\left(t-\tau_{3}\right)$ and $w_{4}\left(t-\tau_{4}\right)$. Therefore, the resulting molecular input signal is represented as

$$
a_{i}\left(t-\tau_{i}\right)=w_{i}\left(t-\tau_{i}\right) * h_{i}(t),
$$

where "**" denotes the convolution operator, $h_{i}(t)$ is a binary sequence that represents the toggle switch activation, modelled by (2) and (3). This sequence is defined as

$$
h_{i}(t)=\left[\begin{array}{c}
s_{1} \\
s_{2} \\
\vdots \\
s_{l}
\end{array}\right],
$$

where $s_{l}$ are either 0 or 1 , for the symbol duration $t_{s}, l=$ $\left\{1, \ldots, t_{f}\right\}$ is the number of sequences and $t_{f}$ is the total length of the system's operation (continuous values).

After being modulated, these molecular input signals will be evaluated by the first layer of the bacteria-based synthetic logic circuit (see Figure $4 b$ ). Each logic gate will then produce a molecular output signal $\left(z_{1}(t)\right.$ for the OR gate and $z_{2}(t)$ for the AND gate) using (4) and (5) and is represented as

$$
\begin{aligned}
\frac{d z_{1}(t)}{d t} & =\frac{a_{1}\left(t-\tau_{1}\right)^{n_{a_{1}}}}{K_{a_{1}}^{n_{a_{1}}}+a_{1}\left(t-\tau_{1}\right)^{n_{a_{1}}}}+\frac{a_{2}\left(t-\tau_{2}\right)^{n_{a_{2}}}}{K_{a_{2}}^{n_{a_{2}}}+a_{2}\left(t-\tau_{2}\right)^{n_{a_{2}}}} \\
& -\gamma_{2} z_{2}(t)+N_{2}(t), \\
& =\frac{\left(w_{1}\left(t-\tau_{1}\right) * h_{1}(t)\right)^{n_{a_{1}}}}{K_{a_{1}}^{n_{a_{1}}}+\left(w_{1}\left(t-\tau_{2}\right) * h_{1}(t)\right)^{n_{a_{1}}}}+ \\
& \frac{\left(w_{2}\left(t-\tau_{2}\right) * h_{2}(t)\right)^{n_{a_{2}}}}{K_{a_{2}}^{n_{a_{2}}}+\left(w_{2}\left(t-\tau_{2}\right) * h_{2}(t)\right)^{n_{a_{2}}}}-\gamma_{1} z_{1}(t)+N_{1}(t),
\end{aligned}
$$




$$
\begin{aligned}
& \frac{d z_{2}(t)}{d t}=\frac{a_{3}\left(t-\tau_{3}\right)^{n_{a_{3}}}}{K_{a_{3}}^{n_{a_{3}}}+a_{3}\left(t-\tau_{3}\right)^{n_{a_{3}}}} \cdot \frac{a_{4}\left(t-\tau_{4}\right)^{n_{a_{4}}}}{K_{a_{4}}^{n_{a_{4}}}+a_{4}\left(t-\tau_{4}\right)^{n_{a_{4}}}} \\
& -\gamma_{2} z_{2}(t)+N_{2}(t), \\
& =\frac{\left(w_{3}\left(t-\tau_{3}\right) * h_{3}(t)\right)^{n_{a_{3}}}}{K_{a_{3}}^{n_{a_{3}}}+\left(w_{3}\left(t-\tau_{3}\right) * h_{3}(t)\right)^{n_{a_{3}}}} \\
& \cdot \frac{\left(w_{4}\left(t-\tau_{4}\right) * h_{4}(t)\right)^{n_{a_{4}}}}{K_{a_{4}}^{n_{a_{4}}}+\left(w_{4}\left(t-\tau_{4}\right) * h_{4}(t)\right)^{n_{a_{4}}}}-\gamma_{2} z_{2}(t)+N_{2}(t) .
\end{aligned}
$$

The molecular output signals are responsible for activating the next layer of the synthetic circuit. Thus, they are diffused from the chambers $\mathrm{A}$ and $\mathrm{B}$ to reach the chamber $\mathrm{C}$, where the last layer of the synthetic circuit is located. The molecules that compose each molecular output signal will travel through the fluidic medium between the chambers independently from each other. The thin membrane that separates the chamber $\mathrm{C}$ from chambers A and B is composed of nanopores allowing only molecular output signals to pass through. This membrane is designed to limit only the bacterial movement [37]. Therefore, the Brownian motion for this system is characterised using the Fick's law of diffusion resulting in the following diffusion channels [51]

$$
\begin{aligned}
& h_{z_{1}}\left(t_{p}\right)=\frac{1}{\sqrt{4 \pi D_{z_{1}}\left(t_{p}\right)}} e^{\frac{-\left\|d_{A C}\right\|^{2}}{4 D_{z_{1}}\left(t_{p}\right)},} \\
& h_{z_{2}}\left(t_{p}\right)=\frac{1}{\sqrt{4 \pi D_{z_{2}}\left(t_{p}\right)}} e^{\frac{-\left\|d_{B C}\right\|^{2}}{4 D_{z_{2}}\left(t_{p}\right)},}
\end{aligned}
$$

where $h_{z_{1}}\left(t_{p}\right)$ and $h_{z_{2}}\left(t_{p}\right)$ are the diffusion channel between chambers $\mathrm{A}$ and $\mathrm{C}$, and between $\mathrm{B}$ and $\mathrm{C}$, respectively; $d_{A C}$ and $d_{B C}$ are the Euclidean distance between chambers $\mathrm{A}$ and $\mathrm{C}$, and between $\mathrm{B}$ and $\mathrm{C}$, respectively; $D_{z_{1}}$ and $D_{z_{2}}$ are the diffusion coefficients for the molecular signals and $t_{p}$ is propagation time inside the bacteria-based synthetic logic circuit. Each bacterium in the population, placed in both chambers $\mathrm{A}$ and $\mathrm{B}$, will produce a molecular output signal concentration that will travel to chamber $\mathrm{C}$. This spatially distributed molecular diffusion will generate a wave that will travel from one chamber to another (from A to C, and B to C). However, as the bacteria are close to each other, we assume that the distances $d_{A C}$ and $d_{B C}$ are between the centres of chambers $\mathrm{A}$ and $\mathrm{C}$, and between the centres of chambers $\mathrm{B}$ and $\mathrm{C}$. Therefore, this will represent the average distance travelled by the total molecular output signal concentration. Furthermore, this definition also affects the propagation time that is assumed to be small enough to travel from one population centre to another and maintain a high molecular concentration.

After being diffused, only a fraction of the molecular output signals $z_{1}(t)$ and $z_{2}(t)$ is able to reach the bacteria population in chamber $C$. Thus, using the results from (9) and (10) and operating them with the diffusion channels described in (11) and (12), we can obtain the fraction of the molecular output signals $z_{1}(t)$ and $z_{2}(t)$, respectively, as

$$
z_{1}^{\prime}(t)=z_{1}(t) * h_{z_{1}}\left(t-\tau_{z_{1}}\right),
$$

and

$$
z_{2}^{\prime}(t)=z_{2}(t) * h_{z_{2}}\left(t-\tau_{z_{2}}\right) .
$$

By substituting (13) and (14) in (5), we are able to finally obtain the molecular output signal $z_{3}(t)$ as

$$
\begin{aligned}
\frac{d z_{3}(t)}{d t} & =\frac{z_{1}^{\prime}(t)^{n_{z_{1}^{\prime}}}}{K_{z_{1}^{\prime}}^{n_{z_{1}^{\prime}}}+z_{1}^{\prime}(t)^{n_{z_{1}^{\prime}}}} \cdot \frac{z_{2}^{\prime}(t)^{n_{z_{2}^{\prime}}}}{K_{z_{2}^{\prime}}^{n_{z_{2}^{\prime}}}+z_{2}^{\prime}(t)^{n_{z_{2}^{\prime}}}} \\
& -\gamma_{3} z_{3}(t)+N_{3}(t), \\
& =\frac{\left(z_{1}(t) * h_{z_{1}}\left(t-\tau_{z_{1}}\right)\right)^{n_{z_{1}^{\prime}}}}{K_{z_{1}^{\prime}}^{n_{z_{1}^{\prime}}}+\left(z_{1}(t) * h_{z_{1}}\left(t-\tau_{z_{1}}\right)\right)^{n_{z_{1}^{\prime}}}} \\
& \cdot \frac{\left(z_{2}(t) * h_{z_{2}}\left(t-\tau_{z_{2}}\right)\right)^{n_{z_{2}^{\prime}}}}{K_{z_{2}^{\prime}}^{n_{z_{2}^{\prime}}}+\left(z_{2}(t) * h_{z_{2}}\left(t-\tau_{z_{2}}\right)\right)^{n_{z_{2}^{\prime}}}}-\gamma_{3} z_{3}(t)+N_{3}(t),
\end{aligned}
$$

\section{Circuit Performance}

We analyse the performance of this end-to-end molecular communications system using two distinct metrics: circuit quality and channel capacity. All the parameters considered for the performance analyses are presented in Table II. We would like to point out that the degradation rates $\gamma_{1}, \gamma_{2}$ and $\gamma_{3}$ values are chosen to be small enough, as well as, the association rates $K_{a_{1}}, K_{a_{2}}, K_{a_{3}}, K_{a_{4}}, K_{z_{1}^{\prime}}$ and $K_{z_{2}^{\prime}}$ are chosen to be high enough so the noise become AWGN and our analysis can be generalised for a wider range of molecular signal frequencies. The engineered bacteria population densities $k_{1}, k_{2}$ and $k_{3}$, as well as, the distances between the chamber compartments centres, $d_{A C}$ and $d_{B C}$, are constrained by the chambers dimensions $(0.1 \times 0.6 \mathrm{~cm})$, which is equivalent to the size of a drug pill.

The total length of the system's operation $t_{f}$, the pulse spread $\eta$, the pulse period $t_{d}$ and the Hill coefficients $n_{a_{1}}$, $n_{a_{2}}, n_{a_{3}}, n_{a_{4}}, n_{z_{1}^{\prime}}, n_{z_{2}^{\prime}}$ are chosen to allow the production of a high molecular signal concentration due to the narrow and well-defined molecular input signal pulses (with proper rise and decay times). The molecular input signals arrays $\rho_{a_{1}}$, $\rho_{a_{2}}, \rho_{a_{3}}$ and $\rho_{a_{4}}$ have 160 samples each. They are processed by the first layer of the circuit within 25 hours, resulting in a new molecular signal arrays $\rho_{z_{1}}, \rho_{z_{2}}$ of 4,000 samples, which are propagated for $t_{p}=0.5$ hours. This will result in higher molecular signal concentration at the centre of the engineered bacteria population placed in compartment $\mathrm{C}$ of the chamber, which will subsequently be processed in the following 25 hours by the last synthetic logic gate, resulting in a molecular output signal of 100,000 samples.

The quality of a system will depend on how it will perform the designed task under certain circumstances [52]. Therefore, to assess the quality of the proposed bacteria-based synthetic circuit, we describe this metric in terms of the accuracy, precision, recall, false positives $(F P)$ and false negatives $(F N)$ rates, for the molecular output signal $z_{3}(t)$. The channel 
TABLE II

PARAMETERS CONSIDERED FOR THIS ANALYSIS

\begin{tabular}{lccc}
\hline Variable & Value & Unit & Reference \\
\hline$k_{1}, k_{2}, k_{3}$ & 10,000 & CFU & $*$ \\
$B_{r}$ & 0.5 & $\mu \mathrm{m}$ & {$[53]$} \\
$d_{A C}, d_{B C}$ & 500 & $\mu \mathrm{m}$ & $*$ \\
$t_{f}$ & 50.5 & hours & $*$ \\
$t_{d}$ & 10 & hours & $*$ \\
$t_{p}$ & 0.5 & hour & $*$ \\
$D_{z_{1}}, D_{z_{2}}$ & 4.9 & $\mathrm{~cm}{ }^{2} / \mathrm{h}$ & {$[54]$} \\
$\gamma_{1}, \gamma_{2}, \gamma_{3}$ & 0.1 & - & $*$ \\
$K_{a_{1}}, K_{a_{2}}, K_{a_{3}}, K_{a_{4}}, K_{z_{1}^{\prime}}, K_{z_{2}^{\prime}}$ & 10 & - & $*$ \\
$n_{a_{1}}, n_{a_{2}}, n_{a_{3}}, n_{a_{4}}, n_{z_{1}^{\prime}}, n_{z_{2}^{\prime}}$ & 2 & - & $*$ \\
$\eta$ & 0.5 & - & $*$ \\
$\rho_{a_{1}}, \rho_{a_{2}}, \rho_{a_{3}}, \rho_{a_{4}}$ & 160 & samples & $*$ \\
$\rho_{z_{1}}, \rho_{z_{2}}$ & 4,000 & samples & $*$ \\
$\rho_{z_{3}}$ & 100,000 & samples & $*$ \\
\hline
\end{tabular}

* Values chosen by the authors.

capacity represents the maximum throughput that the system can achieve under specific conditions.

\section{A. Circuit Quality}

For the first metric, we investigate the impact of the noise and the molecular environmental delay on the production of reliable molecular output signal $z_{3}(t)$. To evaluate the quality of the proposed synthetic circuit, we first define the minimum molecular concentration levels required for the activation of each bacteria-based synthetic logic gate (for a molecular input signal total length of the system's operation $t_{f}$ ). These thresholds are represented as follows.

$$
\begin{aligned}
T_{a_{i}} & =\frac{\max \left(a_{i}(t)\right)}{2}, \\
T_{z_{m}} & =\frac{\max \left(z_{m}(t)\right)}{2},
\end{aligned}
$$

where $a_{i}$ represents each $i=\{1,2,3,4\}$ molecular input signal, and $z_{m}$ represents each $m=\{1,2,3\}$ molecular output signal (see Figure 4).

For all of the analyses presented in this paper, we considered each molecular input signal as an array with $\rho_{a_{i}}$ samples and each molecular output signal as an array with $\rho_{z_{m}}$ samples. These samples can be defined as positive (high level) when it is above the considered threshold and negative (low level) when it is the opposite. The overall quality of the proposed synthetic circuit will depend on the correct identification of the positive and negative molecular output signal samples. In this case, we first define the accuracy_ratio, which is the number of samples that are correctly detected as positive or negative over the total number of samples. This is represented as [52]

$$
\text { accuracy_ratio }=\frac{T P+T N}{T P+T N+F P+F N} \times 100,
$$

where $T P$ and $T N$ are the number of molecular output signal samples that are correctly detected above and below the defined thresholds, respectively; $F P$ and $F N$ are the number of molecular output signal samples that are not correctly detected above and below the defined thresholds, respectively. The precision_ratio is defined as the number of true positive identification over the total positive identifications detected, independent of being either true or false. This is represented as [52],

$$
\text { precision_ratio }=\frac{T P}{T P+F P} \times 100
$$

The recall_ratio or system sensitivity is defined as the number of true positives over the sum of samples that are detected as positive and the ones that should be detected as high level as well. Therefore [52],

$$
\text { recall_ratio }=\frac{T P}{T P+F N} \times 100 .
$$

Using the detected number of false negatives and false positives, we can define the false positive_ratio (FP_ratio) and false negative_ratio (FP_ratio) as [52]

$$
F N \_ \text {ratio }=\frac{F N}{F N+T P} \times 100,
$$

$$
F P \_ \text {ratio }=\frac{F P}{F P+T N} \times 100 .
$$

Using (21), we can also express the recall_ratio as

$$
\text { recall_ratio }=1-F N \_r a t i o .
$$

\section{B. Channel Capacity}

Using the thresholds defined in (16)-(17), we evaluate the channel capacity based on the probabilities of each sample of for the molecular input signals as well as output signal $z_{3}(t)$, and this can be for both high or low levels. Therefore, we define $p_{0}(k)$ and $p_{1}(k)$ as the probabilities of obtaining a low or high (which are represented as 0 or 1) molecular output signals $z_{3}(t)$, respectively. These probabilities are agnostic with respect to the definition of true positives and negatives (including their counterparts). For the channel capacity evaluation, we do not focus on classifying the molecular output signal, but in quantifying the received quantity of low and high molecular output signals from the last gate output. We also define $p_{0}\left(k \mid j_{1}, j_{2}, j_{3}, j_{4}\right)$ and $p_{1}\left(k \mid j_{1}, j_{2}, j_{3}, j_{4}\right)$ as the conditional probabilities of obtaining a high or low level molecular output signal sample $k$ depending on the molecular input signal samples $j_{1}, j_{2}, j_{3}$, and $j_{4}$, where $j_{i} \in\{0,1\}$.

Next, we define the information entropy for the molecular output signal $z_{3}(t)$ as

$$
H\left(z_{3}(t)\right)=-\sum_{k=0}^{\rho_{z_{3}}} p_{0,1}(k) \log _{2} p_{0,1}(k) .
$$

Similarly, the conditional entropy of the molecular output signal $z_{3}(t)$ for molecular input signals $a_{1}(t), a_{2}(t), a_{3}(t)$ 


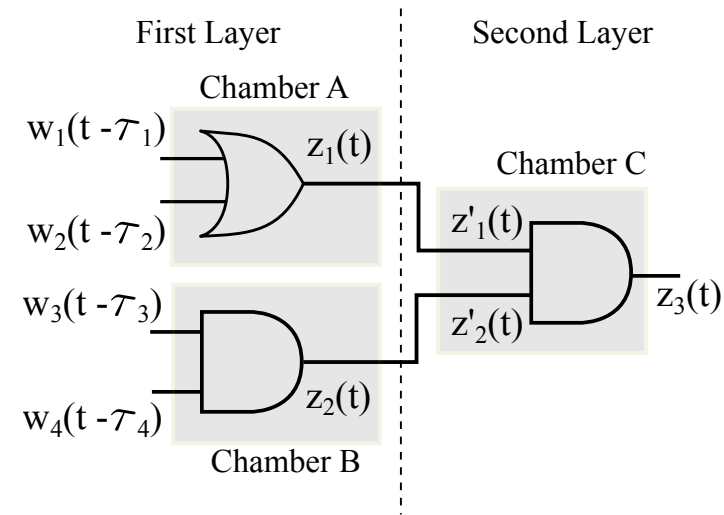

Fig. 5. Representation of the synthetic circuit and the molecular signals considered for this bacteria-based molecular communications system. When the system is not subjected to molecular environmental delays $\left(\tau_{1}=\tau_{2}=\right.$ $\tau_{3}=\tau_{4}=0$ ) the molecular output signal is defined as ideal. For the delayed case, we defined $\tau_{1}=\tau_{3}=0$ and varied the $\tau_{2}$ and $\tau_{4}$ values.

and $a_{4}(t)$ is defined as

$$
\begin{aligned}
& \left.H\left(z_{3} \mid a_{1}(t), a_{2}(t), a_{3}(t), a_{4}(t)\right)\right)= \\
& -\sum_{j_{1}=0}^{\rho_{a_{1}}} \sum_{j_{2}=0}^{\rho_{a_{2}}} \sum_{j_{3}=0}^{\rho_{a_{3}}} \sum_{j_{4}=0}^{\rho_{a_{4}}} p_{0,1}\left(j_{1}, j_{2}, j_{3}, j_{4}\right) \\
& \cdot \sum_{k=0}^{\rho_{z_{3}}} p_{0,1}\left(k \mid\left(j_{1}, j_{2}, j_{3}, j_{4}\right)\right) \cdot \log _{2} p_{0,1}\left(k \mid\left(j_{1}, j_{2}, j_{3}, j_{4}\right)\right) .
\end{aligned}
$$

However, as $p_{0,1}\left(k \mid\left(j_{1}, j_{2}, j_{3}, j_{4}\right)\right)=p_{0,1}(k)$, (25) can be rewritten as

$\left.H\left(z_{3} \mid a_{1}(t), a_{2}(t), a_{3}(t), a_{4}(t)\right)\right)=-\sum_{k=0}^{\rho_{z_{3}}}\left[p_{0,1}(k) \log _{2} p_{0,1}(k)\right]$

$\cdot \sum_{j_{1}=0}^{\rho_{a_{1}}} \sum_{j_{2}=0}^{\rho_{a_{2}}} \sum_{j_{3}=0}^{\rho_{a_{3}}} \sum_{j_{4}=0}^{\rho_{a_{4}}} p_{0,1}\left(j_{1}, j_{2}, j_{3}, j_{4}\right)$.

The channel capacity for this system is defined by the maximum mutual information between the molecular output signal $z_{3}(t)$ and the molecular input signals $a_{1}(t), a_{2}(t), a_{3}(t)$ and $a_{4}(t)$, and is represented as,

$$
\begin{aligned}
& C_{\text {sys }}=\max _{p_{z_{3}(t)}}\left[I\left(z_{3}(t) ; a_{1}(t), a_{2}(t), a_{3}(t), a_{4}(t)\right)\right], \\
& \quad=\max _{p_{z_{3}(t)}}\left[H\left(z_{3}(t)\right)-H\left(z_{3}(t) \mid a_{1}(t), a_{2}(t), a_{3}(t), a_{4}(t)\right)\right] .
\end{aligned}
$$

We use the entropy and conditional entropy values $H\left(z_{3}(t)\right)$ and $H\left(z_{3}(t) \mid a_{1}(t), a_{2}(t), a_{3}(t), a_{4}(t)\right)$ obtained from (24) and
(26) to rewrite (27) as

$$
\begin{aligned}
C_{s y s} & =\max _{p_{z_{3}(t)}}\left[-\sum_{k=0}^{s_{z_{3}}}\left[p_{0,1}(k) \log _{2} p_{0,1}(k)\right]+\right. \\
& \sum_{k=0}^{\rho_{z_{3}}}\left[p_{0,1}(k) \log _{2} p_{0,1}(k)\right] \\
& \left.\cdot \sum_{j_{1}=0}^{\rho_{a_{1}}} \sum_{j_{2}=0}^{\rho_{a_{2}}} \sum_{j_{3}=0}^{\rho_{a_{3}}} \sum_{j_{4}=0}^{\rho_{a_{4}}} p_{0,1}\left(j_{1}, j_{2}, j_{3}, j_{4}\right)\right] .
\end{aligned}
$$

\section{Analytical Results}

In this section, we analyse the operation of the bacteriabased synthetic logic circuit by evaluating its quality and channel capacity. The aim of this analysis is to study the performance of the proposed bacteria-based molecular communications system and define the design parameters required for an accurate operation of the synthetic logic circuit.

Consider a scenario where the system operates under ideal conditions, and this means that the molecular output signal will not suffer from any deterioration in concentration and at the same time the synthetic logic gates of all layers will operate as expected. Figure 5 illustrate the synthetic logic circuit and the molecular signals considered for the proposed system. For our analyses, we considered a synchronised transmission where no molecular environmental delay is present $\left(\tau_{1}=\tau_{2}=\right.$ $\tau_{3}=\tau_{4}=0$ ), and delayed scenarios where we considered two molecular environmental delays as $\tau_{1}=\tau_{3}=0$ and varied the other two $\left(\tau_{2}\right.$ and $\left.\tau_{4}\right)$. Figure 6 presents four plots to illustrate the synchronized and the delayed operation of the proposed bacteria-based synthetic circuit. Figure 6(a) shows the molecular output signals $z_{1}(t)$ and $z_{2}(t)$ when the molecular environmental signals are synchronised with each other (they do not suffer with the molecular environmental delays, $\tau_{1}=\tau_{2}=\tau_{3}=\tau_{4}=0$ ). Figure 6(b) shows the molecular output signals $z_{1}(t)$ and $z_{2}(t)$ when $w_{2}\left(t-\tau_{2}\right)$ and $w_{4}\left(t-\tau_{4}\right)$ are delayed by 5 hours (half of the pulse period, $\left.\tau_{2}=\tau_{4}=t_{p} / 2\right)$. As we can see there are high $z_{1}(t)$ and low $z_{2}(t)$ signals produced when compared to the case depicted in Figure 6(a). IIn general, situations where different molecules are produced from the environment, the molecular environmental signals can arrive delayed into the first layer of the synthetic circuit, and aggregated random system effects can also occur, leading to inaccurate outputs from the circuit. This scenario can be observed by comparing the Figures 6(c) and $6(d)$. The molecular output signal $z_{3}(t)$ obtained from the ideal operation of the circuit, shown in Figure 6(c), is higher than the one produced in a delayed scenario, shown in Figure 6(d). Furthermore, when subjected to molecular environmental delays, the synthetic logic circuit also produced the wrong molecular output signals. Our interest is to investigate further this phenomenon. We analyse different cases in order to define the most appropriate trade-off between the unwanted effects occurring in the first layer of the circuit and the system performance.

In addition to the delayed molecular environmental signals, we also considered the output bit-1 ratio and the gates inputs 


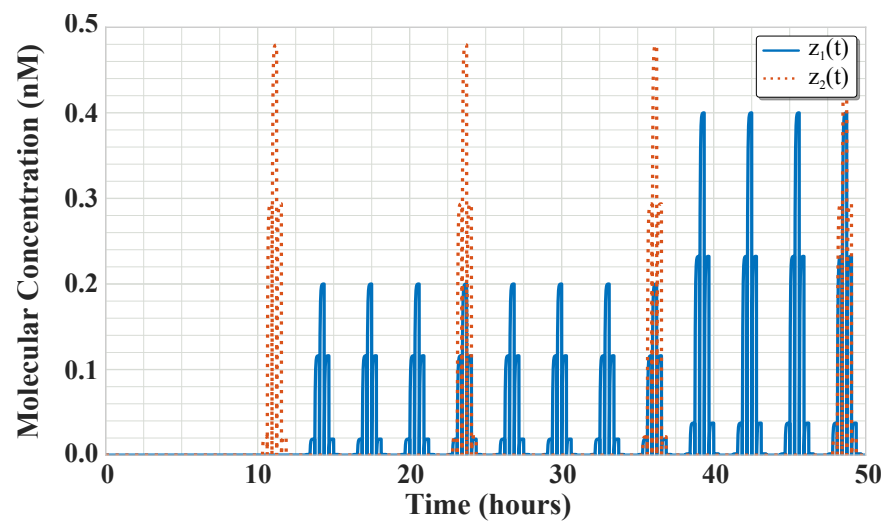

(a) Ideal signal - first layer of the circuit

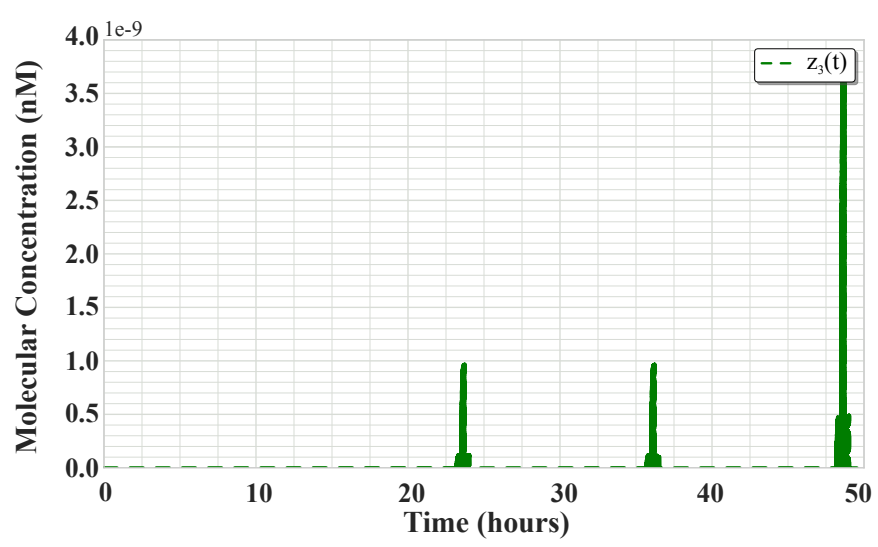

(c) Ideal signal - second layer of the circuit

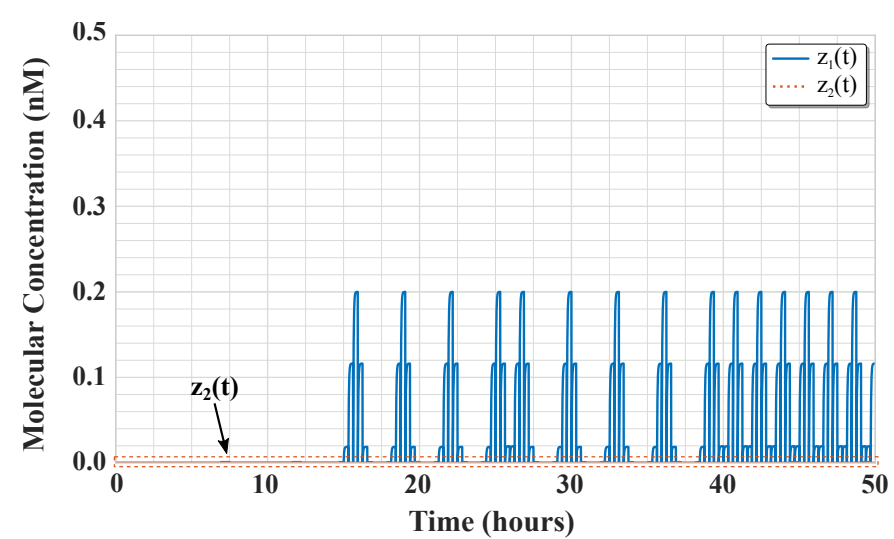

(b) Signals delayed by the half of the pulse period - first layer of the circuit

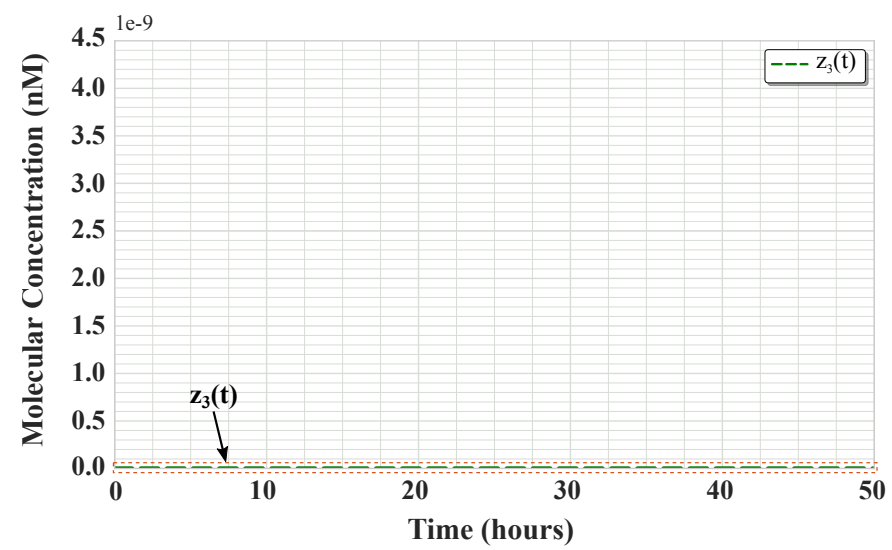

(d) Signals delayed by the half of the pulse period - second layer of the circuit

Fig. 6. Representation of the molecular output signals $z_{1}(t), z_{2}(t)$ and $z_{3}(t)$. For all cases, we considered $h_{i}(t)$ as deterministic sequence of symbols. (a) Representation of the ideal molecular output signals $z_{1}(t)$ and $z_{2}(t)$ from the bacteria-based synthetic logic circuit. (b) When the molecular input signals $w_{2}\left(t-\tau_{2}\right)$ and $w_{4}\left(t-\tau_{4}\right)$ are delayed $\tau_{2}=\tau_{4}=t_{d} / 2$, the molecular output signals $z_{1}(t)$ and $z_{2}(t)$ do not have the same concentration levels of the previous case. (c) As $z_{1}(t)$ and $z_{2}(t)$ are ideal, the molecular output signal $z_{3}(t)$ also will be ideal. (d) Due the molecular environmental delays, the molecular output signal have lower concentration levels than when the system works on an ideal conditions and it produces wrong molecular output signals.

concentration difference as possible unwanted effects for the bacteria-based synthetic logic circuit. If the molecular input signal is composed of a large sequence of bit-1, this excess molecular signals can increase the noise that the bacteria populations are being subjected to, and consequently, can degrade the performance of the communications. A similar effect can occur if a large concentration of molecular input signal arrives at the bacteria population in chambers A and B. Therefore, we focus on these three issues to analyse the performance of the molecular communications of the proposed bacteria-based synthetic logic circuit.

\section{A. Circuit Quality Analysis}

As discussed in the previous sections, conventional digital circuits can have their quality affected if delays are introduced in the system. Therefore, we first investigate the impact caused by a delayed molecular environmental signal on the accuracy, precision, recall, false negative and false positive rates of the circuit.

For this analysis, we define a pulse period $t_{d}=10$ hours, total length of the system's operation $t_{f}=50.5$ hours for the molecular input signals $a_{1}(t), a_{2}(t), a_{3}(t)$ and $a_{4}(t)$; an

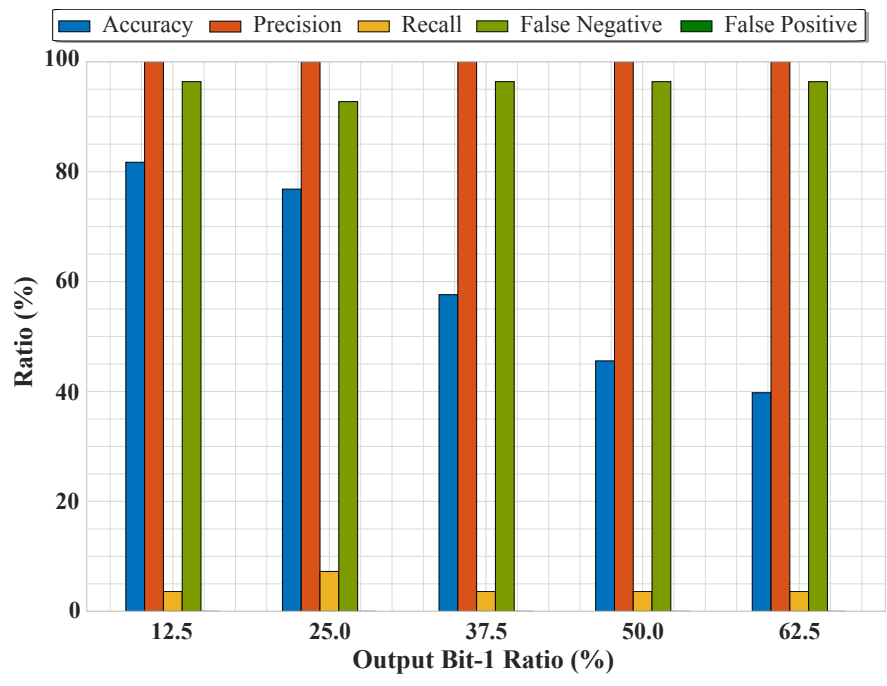

Fig. 7. Quality metrics results for different output bit-1 ratio. For the considered scenario, the circuit worked with $100 \%$ of precision, and no false positive was detected.

AWGN noise $N_{A, B}(t)$ with average $\mu_{N}=0$ and standard deviation of $\sigma_{N}^{A, B}=2 \cdot 10^{-4} \mathrm{nM}$ for the bacteria populations 
located in chambers A and B; a second AWGN noise $N_{C}(t)$ with average $\mu_{N}=0$ and standard deviation of $\sigma_{N}^{C}=2 \cdot 10^{-13}$ $\mathrm{nM}$ for the bacteria population located in chamber $\mathrm{C}$; and we considered the molecular input channels $h_{i}(t)$ as pseudorandom sequences. These noise values were chosen to represent the small fluctuations that can occur during the molecular output signal production [55]. We also considered that two of the molecular environmental signals are delayed by 5 hours (half of the pulse period), and varied the output bit-1 ratio from $12.5 \%$ to $62.5 \%$ of the total number of bits present in the pseudorandom sequences $h_{1}(t), h_{2}(t), h_{3}(t)$ and $h_{4}(t)$. We considered a fixed amplitude for the molecular environmental signals $W_{1}=W_{2}=5 \mathrm{nM}$ and $W_{3}=W_{4}=15 \mathrm{nM}$. No gate input concentration difference was considered for this case.

We can observe in Figure 7 that the accuracy_ratio decreases proportionally with the increase in the output bit-1 ratio. Therefore, higher output bit-1 ratios result in a worse synthetic logic circuit performance. On the other hand, the precision_ratio remained $100 \%$ for all of the considered output bit-1 ratio values, meaning that there is no false positive detected. In the case considered, the recall_ratio remained below $10 \%$, which means that the number of samples wrongly identified as negative is high, and this is based on (20). The FN_ratio value observed from Figure 7 validate this observation, and this is based on (23). Specifically, the circuit misses most of the positive samples, therefore classifying them as negative. This issue is directly related to the threshold definition, and its solution is tied to the selection of a better detection technique. It can be deduced from these results that the output bit-1 ratio is an important unwanted effect for this circuit as it can severely affect the accuracy of the results. In the case of the high false negative ratio, this can be addressed by improving the system reception e.g., this could mean engineering bacteria to be more sensitive to molecules in the last chamber $\mathrm{C}$. Furthermore, the timing that the molecular input signals reach the bacteria populations should be well defined to improve the accuracy of the bacteria-based synthetic logic gates.

\section{B. Communications Analysis}

Channel capacity is an important metric to describe the performance of any communications system. From this perspective, we investigate the effect of the delayed molecular environmental signals as well as the output bit- 1 ratio and the gate input concentration difference on the channel capacity for this bacteria-based molecular communications system. For this analysis, we considered the same AWGN noises from the quality analysis, and the amplitude of the molecular environmental signals as $W_{1}=5 \mathrm{nM}$, a variable amplitude of $W_{2}$ ranging from 5 to $9 \mathrm{nM}, W_{3}=15$, a variable amplitude of $W_{4}$ ranging from 15 to $19 \mathrm{nM}$. The signal amplitudes were selected to enable the investigation of the impact caused on the channel capacity by the gate inputs concentration difference for the two gates placed in the chambers A and B.

We compared the circuit accuracy with the channel capacity to evaluate their relationship. We considered that the molecular input signals are synchronised, and the molecular input

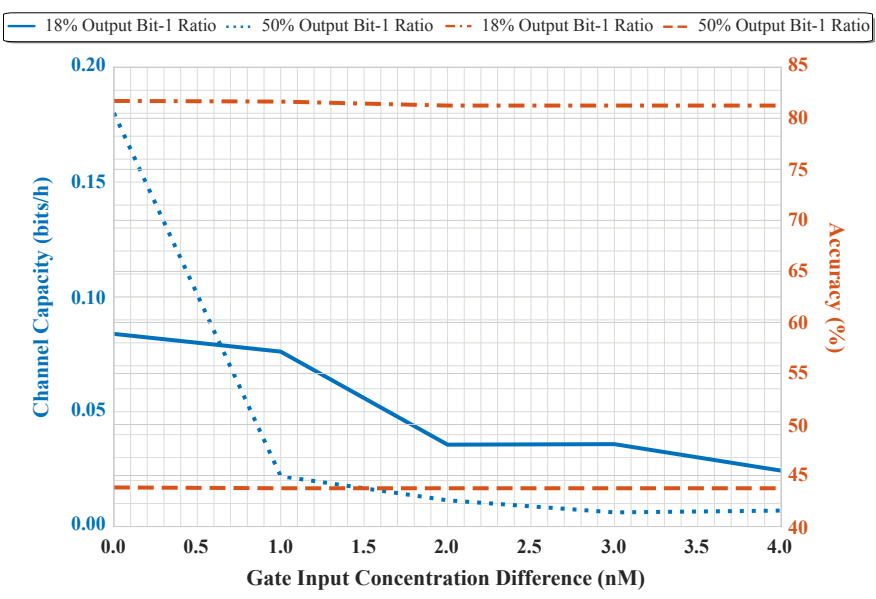

Fig. 8. Evaluation of the channel capacity and circuit accuracy, for a gate inputs concentration difference ranging from 0 to $4 \mathrm{nM}$.

channels $h_{i}(t)$ as random sequences, producing two different output bit-1 ratios (18\% and $50 \%$ ). It can be observed from Figure 8 that the accuracy_ratio, for both molecular input channels, slightly varies with respect to the gate input concentration difference. On the other hand, the channel capacity shows a noticeable variation when the gate input concentration difference and the output bit-1 ratio are increased. For the $18 \%$ output bit-1 ratio (dotted line, see Figure 8), the channel capacity start with a higher value than the $50 \%$ case (solid line, see Figure 8), but stays below 0.02 bits/h after increasing the gate input concentration difference to $1 \mathrm{nM}$. In contrast, the $50 \%$ output bit- 1 ratio reaches this channel capacity value after $4 \mathrm{nM}$. This result suggests the existence of a trade-off between a highly accurate system and a high channel capacity, which is related to the output bit-1 ratio. Therefore, this system design imposes a limitation on the amount of molecular signal that can be accurately processed on the circuit's last gate.

Based on the results presented in Figure 8, we decide to investigate the effect of the molecular environmental delay and the gate inputs concentration difference on the channel capacity when the circuit has an accuracy above $80 \%$. Using (27) and considering a molecular input channel $h_{i}(t)$ that produces a $50 \%$ output bit- 1 ratio, we evaluate the system's channel capacity when there is no delay associated with the molecular environmental signals arriving at the bacteria populations located in chambers $\mathrm{A}$ and $\mathrm{B}$, and when they are delayed by 2 hours $\left(\tau_{2}=\tau_{4}=t_{p} / 5\right)$ and 5 hours $\left(\tau_{2}=\tau_{4}=\right.$ $t_{p} / 2$ ). Figure 9 shows that the channel capacity is inversely proportional to the gate input concentration difference. It also can be noted that for both the synchronised and the 2 hours delay cases, there is a small decrease in the channel capacity when compared to the 5 hours delay case. This result shows that the system can tolerate moderate molecular environmental delays and converge to a steady-state value for the channel capacity. If the proposed bacteria-based synthetic circuit is subjected to a high molecular environmental delay, a buffer or other techniques that will adjust the timing of the molecular input signal might be placed in the chambers $\mathrm{A}$ and $\mathrm{B}$ to counter this unwanted effect.

For our final analysis, we varied the molecular environ- 


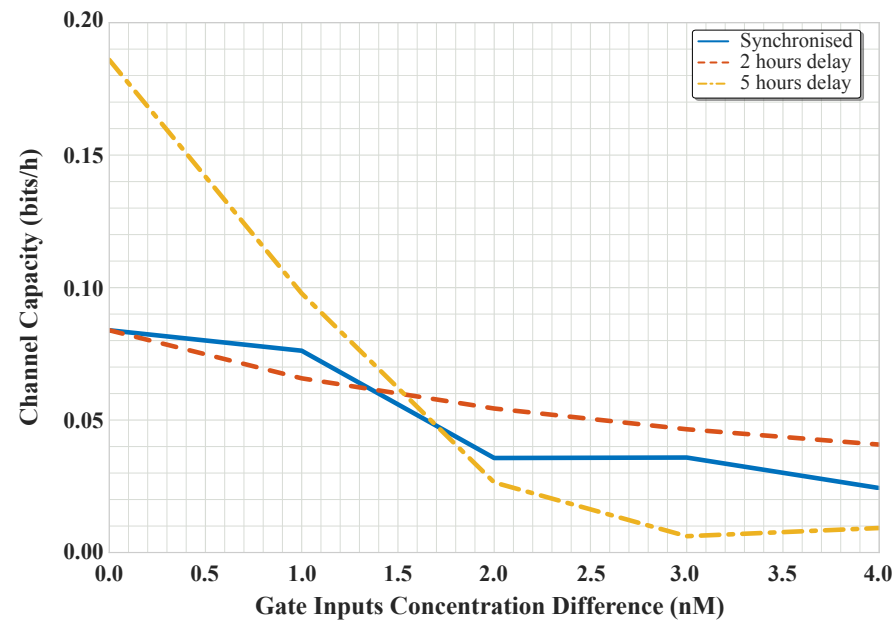

Fig. 9. Evaluation of the channel capacity, for various gate inputs concentration difference for a synchronised and delayed molecular environmental signals.

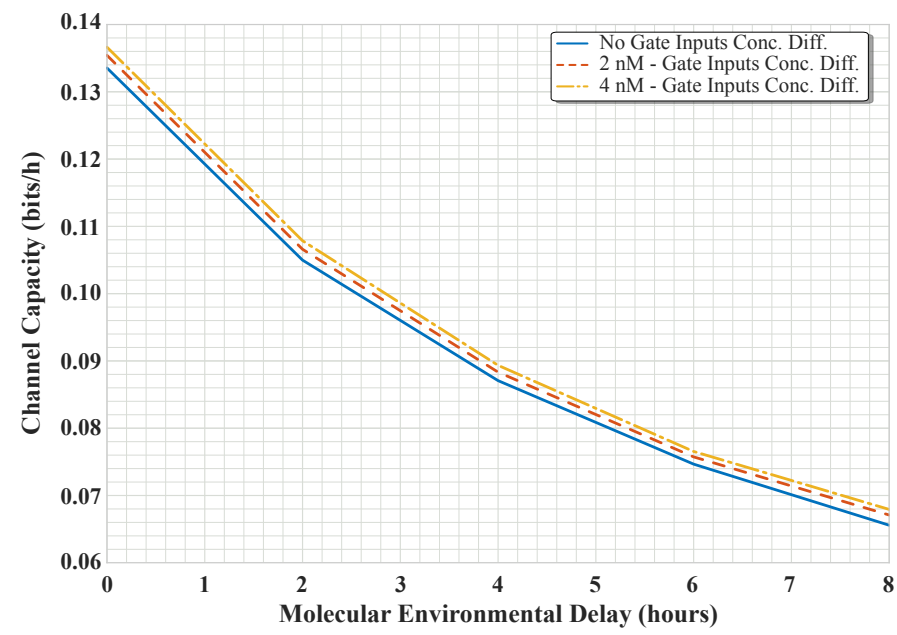

Fig. 10. Channel capacity for different molecular environmental delays, considering three gate inputs concentration difference values.

mental delay, and considered the molecular input channels $h_{i}(t)$ that produces a $50 \%$ output bit-1 ratio, which results in a circuit accuracy of more than $80 \%$. Therefore, Figure 10 shows that the molecular environmental delay can severely affect the channel capacity. On the other hand, greater gate inputs concentration difference result in slightly higher channel capacity. This result corroborates with the result presented in Figure 9 and demonstrates the impact of the gate input concentration difference and the delay on the bacteria-based synthetic logic circuit.

\section{CONCLUSION}

In recent years, new applications have emerged for molecular communications, and in particular through the application of synthetic biology. In this paper, we present the use of molecular communications between bacteria populations that can be used to create synthetic logic circuits. Each population represents a gate, and the communication bus between the gates is established through molecular communications. Given the stochastic property of molecular communications, the paper investigates the quality of the synthetic circuit to determine its accuracy that is dependent on variations of molecular signal input delays, as well as the amplitude of the molecular environmental signals. We also utilized communications theory in terms of channel capacity between the gates and its impact on the quality of the circuit. We found that the molecular concentration difference between the inputs of the circuit can disrupt the operation of the synthetic logic gate, and this can also be affected by the reception thresholds. The molecular concentration difference between the circuit's inputs can impact on the wrong definition in the high and low molecular concentration levels, which can degrade both the quality and channel capacity. Our results suggest that the system can operate with an accuracy above $80 \%$, and the output bit-1 ratio plays a major role in the evaluation of the quality and system performance. These metrics could be further improved by optimising the molecular signal reception on the circuit's last gate. From the obtained results, we can infer that the combination of synthetic biology and molecular communications is an important tool for the design, performance and quality evaluation of bacteria-based synthetic logic circuits.

\section{ACKNOWLEDGMENT}

This work was partially funded by 1) Science Foundation Ireland via the CONNECT research centre (grant no. 13/RC/2077), 2) Science Foundation Ireland via the Vistamilk research centre and 3) Academy of Finland Research Fellow (grant no. 284531).

\section{REFERENCES}

[1] I. F. Akyildiz, J. M. Jornet, and M. Pierobon, "Nanonetworks: A New Frontier in Communications", Communications of the ACM, vol. 54, no. 11 , pp. 84-89, Nov. 2011.

[2] A. Marcone, M. Pierobon, and M. Magarini, "Parity-Check Coding Based on Genetic Circuits for Engineered Molecular Communication Between Biological Cells," IEEE Transactions on Communications, vol. 66, no. 12, pp. 6221-6236, Dec. 2018.

[3] J. R. Porter and E. Batchelor, "Using Computational Modeling and Experimental Synthetic Perturbations to Probe Biological Circuits," in Computational Methods in Synthetic Biology, ser. Methods in Molecular Biology, M. A. Marchisio, Ed. New York, NY: Springer New York, 2015, vol. 1244, pp. 259-276.

[4] C. Sabu et al., "Bioinspired and biomimetic systems for advanced drug and gene delivery," Journal of Controlled Release, vol. 287, no. June, pp. 142-155, Aug. 2018.

[5] R. Sarpeshkar, "Analog synthetic biology," Philosophical Transactions of the Royal Society A: Mathematical, Physical and Engineering Sciences, vol. 372, no. 2012, pp. $20130110-20130110$, Feb. 2014.

[6] D. T. Riglar and P. A. Silver, "Engineering bacteria for diagnostic and therapeutic applications," Nature Reviews Microbiology, vol. 16, no. 4, pp. 214-225, Feb. 2018.

[7] Y. Higashikuni, W. C. Chen, and T. K. Lu, "Advancing therapeutic applications of synthetic gene circuits," Current Opinion in Biotechnology, vol. 47, pp. 133-141, Aug. 2017.

[8] H. K. Allen et al., "Finding alternatives to antibiotics," Annals of the New York Academy of Sciences, vol. 1323, no. 1, pp. 91-100, sep 2014.

[9] P. Hillenbrand, G. Fritz, and U. Gerland, "Biological Signal Processing with a Genetic Toggle Switch," PLoS ONE, vol. 8, no. 7, p. e68345, Jul. 2013.

[10] B. Saltepe et al., "Cellular Biosensors with Engineered Genetic Circuits," ACS Sensors, vol. 3, no. 1, pp. 13-26, Nov. 2018.

[11] J. J. Collins, T. S. Gardner, and C. R. Cantor, "Construction of a genetic toggle switch in Escherichia coli," Nature, vol. 403, no. 6767, pp. 339342, Jan. 2000. 
[12] N. Dalchau et al., "Computing with biological switches and clocks," Natural Computing, vol. 17, no. 4, pp. 761-779, June 2018.

[13] A. Urrios et al., "Plug-and-Play Multicellular Circuits with TimeDependent Dynamic Responses," ACS Synthetic Biology, vol. 7, no. 4, pp. 1095-1104, Mar. 2018.

[14] A. Sengupta, H. B. Pakrasi, and P. P. Wangikar, "Recent advances in synthetic biology of cyanobacteria," Applied Microbiology and Biotechnology, pp. 1-15, May 2018.

[15] A. Tamsir, J. J. Tabor, and C. A. Voigt, "Robust multicellular computing using genetically encoded NOR gates and chemical 'wires",, Nature, vol. 469, no. 7329, pp. 212-215, Jan. 2011.

[16] T. Nakano, A. W. Eckford, and T. Haraguchi, "Molecular communication", Cambridge University Press, 2013.

[17] L. Felicetti et al., "Applications of molecular communications to medicine: A survey," Nano Communication Networks, vol. 7, pp. 27-45, Mar. 2016.

[18] O. B. Akan et al.,"Fundamentals of molecular information and communication science",Proceedings of the IEEE, vol. 105, no. 2, pp. 306-318, Feb. 2017.

[19] M. T. Barros, "Ca2+-signaling-based molecular communication systems: Design and future research directions," Nano Communication Networks, vol. 11, pp. 103-113, Mar. 2017.

[20] Y. Chahibi, "Molecular communication for drug delivery systems: A survey," Nano Communication Networks, vol. 11, pp. 90-102, Mar. 2017.

[21] V. Loscrì, A. M. Vegni, and G. Fortino, "On the Interaction between a Nanoparticulate System and the Human Body in Body Area Nanonetworks," Micromachines, vol. 6, no. 9, pp. 1213-1235, Aug. 2015.

[22] B. Atakan, O. B. Akan, and S. Balasubramaniam, "Body area nanonetworks with molecular communications in nanomedicine", IEEE Communications Magazine, vol. 50, no. 1, pp. 28-34, Jan. 2012.

[23] T. Nakano et al., "Molecular Communication and Networking: Opportunities and Challenges," IEEE Transactions on NanoBioscience, vol. 11, no. 2, pp. 135-148, Jun. 2012.

[24] G. Wei and R. Marculescu, "Miniature Devices in the Wild: Modeling Molecular Communication in Complex Extracellular Spaces," IEEE Journal on Selected Areas in Communications, vol. 32, no. 12, pp. 2344-2353, Dec. 2014.

[25] A. O. Bicen, J. J. Lehtomäki, and I. F. Akyildiz, "Shannon Meets Fick on the Microfluidic Channel: Diffusion Limit to Sum Broadcast Capacity for Molecular Communication," IEEE Transactions on NanoBioscience, vol. 17 , no. 1, pp. 88-94, 2018.

[26] L. Felicetti et al., "TCP-like molecular communications", IEEE Journal on Selected Areas in Communications, vol. 32, no. 12, pp. 2354-2367, Dec. 2014.

[27] V. Loscrì, L. Matekovits, I. Peter, and A. M. Vegni, "In-body Network Biomedical Applications: from Modeling to Experimentation,", IEEE Transaction on Nanobioscience, vol. 15, no. 1, pp. 53-61, Jan. 2016.

[28] L. C. Cobo and I. F. Akyildiz, "Bacteria-based communication in nanonetworks," Nano Communication Networks, vol. 1, no. 4, pp. 244256, Dec. 2010.

[29] A. Einolghozati, M. Sardari, and F. Fekri, "Design and Analysis of Wireless Communication Systems Using Diffusion-Based Molecular Communication Among Bacteria," IEEE Transactions on Wireless Communications, vol. 12, no. 12, pp. 6096-6105, Dec. 2013.

[30] D. P. Martins et al., "Molecular Communications Pulse-Based Jamming Model for Bacterial Biofilm Suppression," IEEE Transactions on NanoBioscience, vol. 17, no. 4, pp. 533-542, Oct. 2018.

[31] D. Ausländer et al., "Programmable full-adder computations in communicating three-dimensional cell cultures," Nature Methods, vol. 15, no. 1, p. 57, Dec. 2018.

[32] Z. Li et al.,"Bacteria-based AND logic gate: a decision-making and self-powered biosensor", Chemical Communications, vol. 47, no. 11, pp. 3060-3062, Mar. 2011.

[33] K. Kim et al.,"Logic circuit upon angiogenic response controlled by enzyme-linked iron oxide microparticles-towards biocomputing in human cells", Chemical Communications, vol. 48, no. 55, pp. 6918-6920, May 2012.

[34] Y. Hu et al., "Programming the quorum sensing-based AND gate in Shewanella oneidensis for logic gated-microbial fuel cells",Chemical Communications, vol. 51, no. 20, pp. 4814-4817, Feb. 2015.

[35] J. Qian, S. Pullela, and L. Pillage, "Modeling the "Effective capacitance" for the RC interconnect of CMOS gates," IEEE Transactions on Computer-Aided Design of Integrated Circuits and Systems, vol. 13, no. 12, pp. 1526-1535, Dec. 1994.

[36] F. Dartu, N. Menezes, and L. Pileggi, "Performance computation for precharacterized CMOS gates with RC loads," IEEE Transactions on
Computer-Aided Design of Integrated Circuits and Systems, vol. 15, no. 5 , pp. 544-553, May 1996.

[37] J. Shim et al., "Controlling the contents of microdroplets by exploiting the permeability of PDMS," Lab on a Chip, vol. 11, no. 6, p. 1132, Mar. 2011.

[38] D. E. Contois, "Kinetics of Bacterial Growth: Relationship between Population Density and Specific Growth Rate of Continuous Cultures",Microbiology, vol. 21, no. 1, pp. 40-50, Aug. 1959.

[39] C. C. Spicer, "The Theory of Bacterial Constant Growth Apparatus", Biometrics, vol. 11, no. 2, pp. 225-230, June 1955.

[40] M. Pierobon and I. F. Akyildiz, "A Physical End-to-End Model for Molecular Communication in Nanonetworks," IEEE Journal on Selected Areas in Communications, vol. 28, pp. 602-611, May 2010.

[41] T. Sakurai, "Closed-Form Expressions for Interconnection Delay, Coupling, and Crosstalk in VLSI's," IEEE Transactions on Electron Devices, vol. 40, no. 1, pp. 118-124, Jan. 1993.

[42] S. Krishnaswamy et al., "Probabilistic transfer matrices in symbolic reliability analysis of logic circuits," ACM Transactions on Design Automation of Electronic Systems, vol. 13, no. 1, pp. 1-35, Jan. 2008.

[43] P. Mehta et al., "Information processing and signal integration in bacterial quorum sensing," Molecular Systems Biology, vol. 5, no. 1, p. 325, Jan. 2009.

[44] W. K. Purves et al., Life: The Science of Biology. Macmillan, 2001.

[45] M. Kuscu and O. B. Akan, "Maximum Likelihood Detection With Ligand Receptors for Diffusion-Based Molecular Communications in Internet of Bio-Nano Things," IEEE Transactions on NanoBioscience, vol. 17 , no. 1 , pp. 44-54, Jan. 2018

[46] M. Pierobon and I. F. Akyildiz, "Noise Analysis in Ligand-Binding Reception for Molecular Communication in Nanonetworks," IEEE Transactions on Signal Processing, vol. 59, no. 9, pp. 4168-4182, 2011.

[47] J. Bonnet et al., "Amplifying Genetic Logic Gates," Science, vol. 340, no. 6132, pp. 599-603, May 2013.

[48] C. D. Cox et al., "Analysis of noise in quorum sensing," Omics: a journal of integrative biology, vol. 7, no. 3, pp. 317-34, Sep. 2003.

[49] M. L. Simpson et al., "Frequency domain analysis of noise in autoregulated gene circuits", Proceedings of the National Academy of Sciences, vol. 100, no. 8, pp. 4551-4556, Apr. 2003.

[50] A. Papoulis, and S. Unnikrishna Pillai, "Probability, random variables, and stochastic processes", Tata McGraw-Hill Education, 2002.

[51] I. Llatser et al., "N3Sim: Simulation framework for diffusion-based molecular communication nanonetworks," Simulation Modelling Practice and Theory, vol. 42, pp. 210-222, Dec. 2013.

[52] D. L. Olson and D. Delen, Advanced Data Mining Techniques. Springer Science \& Business Media, 2008

[53] W. R. Schneider and N. R. Doetsch, "Effect of Viscosity on Bacterial Motility," Journal of Bacteriology, vol. 117, no. 2, pp. 696-701, Feb. 1974.

[54] P. Melke et al., "A Cell-Based Model for Quorum Sensing in Heterogeneous Bacterial Colonies," PLoS Computational Biology, vol. 6, no. 6, pp. 1-13, June 2010 .

[55] M. Thattai and A. van Oudenaarden,"Intrisic noise in gene regulatory networks", Proceedings of the National Academy of Science, vol. 98 , no. 15, pp. 8614-8619, July 2001 